\title{
SOBRE EL CONCEPTO DE PARÁMETRO EN LA «THEORY OF GOVERNMENT AND BINDING»*
}

Pedro Santana Martinez

Universidad de La Rioja

\section{Planteamiento}

En este artículo vamos a tomar un concepto central de una teoría gramatical, la llamada de la rección y el ligamiento, que, fundada por Chomsky, ha sido mantenida por éste y por numerosos seguidores a lo largo de los años ochenta. Lo que nos interesa es estudiar el compromiso entre ese concepto clave (el de parámetro) y las corroboraciones empíricas de la teoría. Nuestro estudio, por tanto, tiene un carácter metateórico, o si se quiere propio incluso de la filosofía de la ciencia, de la filosofia de la lingüística en este caso. Sin embargo, el origen particular del mismo se halla en trabajos de carácter empírico, en la medición de incrementos de la competencia gramatical en aprendices del inglés como segunda lengua. Para la realización de tales estudios, habíamos adoptado la Gramática Generativa en la versión de los años ochenta como «marco teórico de referencia», esto es, al menos las descripciones gramaticales de las estructuras implicadas se realizaban según los dictados de tal teoría. Pero además, nos encontrábamos con que determinados presupuestos centrales

* Trabajo realizado con financiación de la DGICYT. Núm. de Referencia del Proyecto PB92-0757. 
de la teoría no eran adecuados para la constitución de una teoría que fuera no ya falsable por utilizar el término popperiano, sino ni siquiera verificable en el sentido de que los términos teóricos de las «ciencias gramaticales» no hallan una correspondencia con clases bien especificadas de datos observacionales. $Y$ si se quiere evitar el situarse dentro de las teorías de la ciencia más o menos cercanamente evocadas con las palabras anteriores, déjenos el lector repetir la misma idea diciendo que el problema para la lingüística es triple pues sus datos son de naturaleza fenoménica, esto es, son evidencias para un sujeto que puede ser el mismo hablante, pero no poseen ninguna objetividad fisicalista pertinente para las categorías lingǘsticas. Además la superación de un nivel fenoménico de descripción se encuentra con la dificultad de que los conceptos de la lingǘrstica o son muy generales $y$, hasta cierto punto, vacíos en cuanto conceptos linguísticos ${ }^{1}$, o tienen una validez descriptiva relativamente reducida y son diff́cilmente extensibles interlinguísticamente.

El artículo que sigue, pues, ha de entenderse como un intento programático encaminado a recuperar para la teoría una mayor superficie de contacto y una mayor variedad de relaciones con la contrastación empírica.

La teoria de la rección y el ligamiento (Theory of Government and Binding, GBT) se resume en los dos puntos siguientes, aunque de manera abusivamente sucinta:

1) Existe una Gramática Universal (GU) que es conocida —en algún sentido de esta palabra - por todo ser humano al nacer. Esta GU consta de principios y de los llamados parámetros. Estos pueden tomar dos o más valores, lo que tiene lugar al aprender una lengua determinada. La GU, esto es, los principios y los parámetros no regulan todo el sistema y todos los usos de una lengua real, sólo lo que se llama su núcleo.

Muchos fenómenos lingüísticos corresponden a la llamada periferia ${ }^{2}$. Por otro lado, la simple consulta de la literatura generativista de los ochenta pone de manifiesto cómo la GBT se ha centrado preferentemente en la sintaxis y a ella, sobre todo, se refieren los parámetros que han sido definidos ${ }^{3}$.

1 Es el caso, por ejemplo, de la Gramática Universal, concepto que puede ser pertinente desde el punto de vista psicológico, tal vez antropológico, etc., pero no es propiamente un concepto gramatical de ninguna gramática particular. VEase más abajo qué tratamiento damos al concepto en la sección 3.

2 Se habla frecuentemente de periferia marcada, siendo el núcleo lo no marcado. La distinción marcado / no marcado se retoma en la GBT de tal manera que se confunde con la distinción entre núcleo y periferia, lo que parece por lo menos problemático.

${ }^{3}$ Sin embargo, según el llamado Lexical Parameterization Principle, los valores de los parámetros serian clases de unidades léxicas o de otro tipo de unidades. Véase Manzini (1993), un trabajo al que tendremos ocasión de referimos más adelante, para una aplicación el principio. 
2) La descripción gramatical de una lengua, su gramática nuclear particular, y también la G.U. tienen una estructura modular. La descripción de una oración se hace teniendo en cuenta todos los módulos que forman la gramática. Es pertinente recordar aquí que la modularidad de la facultad linguística se utiliza para probar la modularidad de la mente o de la cognición en general. Un componente de la GBT, la teoría de la X con barra, facilita un sistema de marcado de las categorías sintácticas en la estructura oracional y, al tiempo, da pie para teorizar sobre la fundamental similitud formal de todas las clases de sintagmas y unidades superiores al sintagma. Sobre el carácter real de esta teoría, sobre si ésta es sólo un procedimiento de etiquetado mera «botánica verbal», o es algo más, se ha producido alguna polémica. Por ejemplo, Kornai \& Pullum (1990) sólo conceden «very little substance in its claim [el de la teoría de la X con barra]» (24). Estos autores eliminan de la teoría la noción de barra - lo que significa que las llamadas proyecciones no tienen ningún valor teórico, esto es, no limitan las estructuras de frase posibles-y la reconstruyen sobre la noción, para ellos fundamental, de cabeza, inglés "head". Mencionamos esto porque en nuestra argumentación será central la cuestión de la constitución de la clase general de sintagma (o frase, ambos traducen en estos contextos el ingles " $p h$ rase"). En este sentido, aunque la teoría ortodoxa sea sometida a un fuerte cuestionamiento, hay un elemento de la misma, el recién mencionado de la construcción del concepto o clase general de sintagma, que se ha convertido en supuesto ubicuo. Y esto a tal punto que la teoría de la X con barra se ha acoplado a otras propuestas gramaticales muy diversas. Véase, por ejemplo, Sells (1985).

Además de lo dicho, en la teoría se afirma que cada oración posee diferentes niveles de representación, todos ellos necesarios: estructura-d, estructura-s, forma lógica y forma fonética.

Si el segundo punto alude metagramaticalmente a cómo se estructura la teorfa y, lo que es un correlato habitual a lo largo de la historia de la gramática generativa chomskiana, a cómo se estructura en una supuesta mente/cerebro en expresión usual en la literatura generativista, que intenta rendir pleitesía al tiempo al cognitivismo y a la neurología-, el primero es especialmente pertinente en lo que hace a los procesos de adquisición y aprendizaje. En efecto, parece que el infante dispone de un sistema de posibilidades, de potencias o facultades, que se actualizan al aprender una lengua determinada, justamente su lengua materna. Nadie habla la GU, ésta no es un idioma. Ahora bien, el proceso de aprendizaje en lo que hace al núcleo de la lengua consiste en la adjudicación de unos valores, dentro de un dominio prefijado, a los parámetros.

Puede comprenderse que este modelo de adquisición ha de resultar satisfactorio cuando se sostiene que el estímulo que recibe un niño es insuficiente 
para aprender una lengua. Es el llamado por Chomsky problema de Platón. Como se sabe, la trayectoria científica de aquél puede caracterizarse, entre otras cosas, por una continua oposición al conductismo, al que critica entre otras cosas porque no puede aportar un modelo verosímil de aprendizaje que explique cómo aprendemos una primera lengua, dado el bajo nivel y cantidad de estímulos a que estamos expuestos en proporción a lo que acabamos por aprender, todo esto siempre según Chomsky.

Aunque el y otros autores postulan un número relativamente pequeño de parámetros - quince, tal vez veinte- no se encuentra en la literatura mención más que de cinco o seis 4 .

De una manera vulgar, pero reveladora, podría decirse que cada parámetro controla-no en el sentido de la teoría del control, uno de los módulos de la GBT, sino en un sentido general-, regula o se corresponde con varios fenómenos lingüisticos, evidentes estructuralmente en la mayoría de los casos. La palabra «fenómeno» lleva una carga semántica demasiado comprometedora, pero nos parece expresivo este modo de hablar y la utiliza el mismo Chomsky. Así en Chomsky (1981), las ya famosas Lecciones de Pisa, dice:

Languages may select from among the devices of UG, setting the parameters in one or another way, to provide for such general processes as those that were considered to be specific rules in earlier work. At the same time phenomena that appear to be related may prove to arise from the interaction of several components, some shared, accounting for the similarity. (7)

Y más adelante, dentro todavía del primer capítulo de la obra, ' Outline of the theory of core grammar':

It has, for example, proven quite fruitful to explore redundancies in grammatical theory, that is, cases in which phenomena are overdetermined by a given theory ... (13-14)

Y algo más abajo el término «phenomenon» en singular, «phenomena» en plural, aparece más veces, en ocasiones alternando con «facts». Es frecuente, en el caso de la sintaxis, hablar también de «construcciones». La cuestión que resulta aquí pertinente es la de si el uso del primer término es, por así decir,

4 Sucede que el recuento de parámetros mencionado en la literatura varfa por cuanto, como tendremos ocasión de ver, se considera a veces la presencia de varios paránetros donde otros autores han procedido a una unificación. 
inocente o si más bien nos pone en la pista de alguna característica fundamental del enfoque propio de la GBT.

Se diría que los datos lingǘsticos describibles estructuralmente corresponden a un nivel fenoménico, se manifiestan ante la conciencia del sujeto hablante. A este respecto se ha sostenido que el estructuralismo praguense - y no es esa la única orientación lingüística europea que se ampara bajo tal fundamentación - halla su «transfondo filosófico»' en la fenomenología. En este sentido, los principios abstractos a los que la GBT pretende llegar podrían verse no sólo como leyes generales, sino también como superaciones de un estadio fenoménico de la ciencia del lenguaje.

Ahora bien, el tipo de objetividad que la GBT (y la lingüística y las ciencias humanas en general) pretende se escapa de cualquier control fisicalista. Queremos decir que, por ejemplo, las categorías que utiliza el gramático se alejan enormemente de las categorías extragramaticales que permiten analizar la superficie de la cadena hablada - lo que sería grosso modo cierto de la fonología praguense- $y$, desde el punto de vista de los fenómenos, se alejan también de las mismas intuiciones del hablante. En otros términos y también por vía de ejemplo. No existe un nexo sistemático entre descripción física de unos sonidos articulados y los niveles más bajos de descripción o análisis fonológicos, por no mencionar otros niveles de representación. Se diría, en terminología tomada de la tagmémica, que el hiato entre la perspectiva etic y la emic es insalvable. El problema gnoseológico que aquí asoma es, creemos, el de si los principios de la teorfa convierten a ésta en completa -completa en lo que hace al núcleo, a la "core grammar", cuya delimitación es también problemática-y si los principios no son soluciones ad hoc para solucionar casos particulares ${ }^{6}$.

3 Ver López García (1989: 42 y ss.), tanto para un somero repaso de la cuestión como para una vindicación de la fundamentación fenomenológica de una determinada teoría lingúrstica.

- La primera cita que hicimos de Chomsky señala implícitamente a la cuestión de la completitud de la teorla gramatical. Si los fenómenos pueden surgir, en su variedad, de la interacción de varios componentes abstractos lo más necesario cientúficamente es cerrar la lista de esos componentes y no recurrir a ninguna 'virtus grammaticalis' adecuada al caso a resolver. Véase sobre esto infra en este mismo artículo. Por otro lado, la no recursividad de las gramáticas en sus zonas periféricas es reconocida por Chomsky desde, al menos, Chomsky ( 1981). En Chomsky ( 1991 ) es patente un sesgo no del todo disímil a la posición que expresamos: «While there is no clear sense to the idea that language is 'designed for use' or 'well-adapted to its functions', we do expect to find close links between the properties of the language and the manner of its use.» Esto es, el korgano» biologico que es el lenguaje está sujeto a las inadecuaciones consustanciales a la contingencia de la evolución biológica. No es una facultad ni un sistema perfectos, pero no puede ignorarse su función y su uso. 
Antes de exponer el asunto de manera más técnica, y dado que la literatura de la GBT no abunda en el estudio más que de unas pocas lenguas, nos permitimos las siguientes puntualizaciones y comentarios, formulados deliberadamente en este lenguaje que opone fenómenos y principios:

- Los parámetros son los mismos en todas y cada lengua. Es éste un presupuesto irrenunciable de la teoría porque se parte de un GU común.

- Un parámetro dado controla los mismos fenómenos en todas las lenguas. Esto plantea un serio problema a la lingüística descriptiva. En una palabra, exige una aparato de categorías gramaticales descriptivas con una capacidad interlinguística total.

- La seguridad de que el número de valores que puede adoptar un parámetro dado se conoce. Permítasenos un ejemplo. Se postula la existencia de un parámetro que rige la dirección principal de ramificación: Dentro de un sintagma cualquiera, si su cabeza está delante o detrás, a la izquierda o a la derecha, según el modo de hablar dictado por nuestro sistema de escritura. Esto indicaría dos valores. Podemos, sin embargo, pensar en una lengua que admita, sin que tal cosa separara una forma marcada de una no marcada, la posibilidad absolutamente indeterminada de ramificación a la derecha y a la izquierda. Tres serían los valores entonces para el parámetro. Que tal lengua se halle de hecho excluida del universo de las lenguas realmente existentes, valga la expresión, debería comprobarse empíricamente. En caso contrario, la GU debería reformularse.

\section{Alcance de los parámetros en la GBT}

Vamos a replantear la cuestión del alcance de cada parámetro, esto es, del dominio de fenómenos al que se aplica. En algunos casos la formulación de una parámetro es de una simplicidad ejemplar. En Cook (1988) se define el head parameter asi:

A language has the heads on the same side in all its phrases. (9)

Esto es, delante o detrás. Veamos qué primera definición se da en la misma obra del pro-drop parameter:

(It is) a parameter with two settings

either INFL is a proper governor

or INFL is not a proper governor (42)

Ahora bien, habría una primera definición, menos técnica, implícita en una formulación inicial como «the pro-drop parameter concerns wether a language 
has declarative sentences without apparent subjects, known as null subject or subjectless sentences» (Cook: 1988,38). Quizá convenga recordar que pro es una de las cuatro categorías vacías de las que habla la GBT, entidades no representadas en la forma fonética. Governing, traducible por 'rección', es una cierta relación entre dos elementos de una estructura sintáctica. En Rizzi (1986), la formulación se hace más comprensiva. Tal como se expone en Haegeman (1991), el parámetro en cuestión es:

The prodrop parameter

prop is governed by $X^{0}$

$y$ Let $X$ be the licensing head of an occurrence of pro: then pro has the grammatical specification of the features on $X$ co-indexed with it. (418)

No necesitamos entrar en el análisis de la cuestión. Una cabal comprensión del texto citado requiere un trabajo serio de familiarización con la teoría. Sin embargo, lo que queremos subrayar puede verse claramente: el dominio del parámetro gana generalidad de una formulación a otra ${ }^{7}$. Y gana también abstracción. Pero también se diría: a priori ganan las posibilidades de que el número de valores asignables al parámetro sea mayor de dos.

Volvamos al head parameter. La simplicidad de la formulación citada es tan sólo aparente. De hecho, cuatro son los tipos distintos de sintagmas (phrases) que distingue la GBT. ¿Por qué a priori se debían comportar las heads, los núcleos o cabezas, de todos los sintagmas de la misma forma? Pero incluso en formulaciones más restringidas que la citada al respecto de ese parámetro o de otro cualquiera de los parámetros hay dificultades. Por ejemplo en Fanselow ed. (1993), tomando un módulo de la teoría, el de Binding o ligamento, se dice que:

Principles A and B of the Binding Theory require that anaphors be bound and pronouns be free in a certain local domain $\Sigma$ :, while parameters constrain the range of variation for $\Sigma: \ldots$ (VIII)

7 El lector puede pensar que todas las construcciones que regula el parámetro en cuestión se refiere a la presencia o no de sujeto delante del verbo, pero, como veremos más adelante, se registra una variabilidad interlingüística tal que no permite distinguir entre lenguas pro-drop y lenguas no pro-drop sin más. Por otro lado, el dominio de aplicación del llamado Principio de la Categoría Vacía puede extenderse bastante naturalmente a fenómenos en que interviene la riqueza flexiva de la lengua con respecto al orden de palabras. Piénsese en que las lenguas de gran riqueza flexiva permiten la alteración del orden de los componentes de la cadena-s en muchos más casos que el de sujeto verbo. Si el valor del parámetro pro-drop tiene que ver con la concordancia, podemos aventurar razonablemente cabezas similares a INF en otros lugares de la estructura gramatical no sólo en los que se refieren a la concordancia sujeto-verbo. Así, el dominio de este param En cualquier caso remitimos al lector a la discusión ulterior. 
Dejemos, una vez más si se nos permite, el detalle de la cuestión. Lo que hace Fanselow es pasar a discutir esta vision ortodoxa, pero al hacerlo parece cerrar los ojos a ciertas evidencias, lo que a su vez nos recuerda el difícil estatuto de los «hechos», los fenómenos y la superación de éstos en una teoría no meramente fenómenica en las ciencias del lenguaje. Fanselow sostiene que postular el parámetro no es decir nada de la UG o, más bien, que ésta no dice nada sobre el asunto de la dirección de la asignación de caso. Sin embargo, en una nota a pie de página comenta - y el asunto lo trata Zimmmermann en el artículo que firma dentro de Fanselow ( 1993) - que los sintagmas prepositivos podrían no ser unidireccionales. Recuerda que preposiciones alemanas como entlang y wegen pueden aceptar complementos a los dos lados. Ahora bien, nos tememos que es éste un caso típico de eclipse de las realidades lingüísticas, y si lo citamos es a efectos de hacer ver cómo ciertos análisis gramaticales parecen desarrollarse gracias a cierto desvío de la atención. Para empezar cabría decir que 'preposición' es término que pide el principio. De hecho, es habitual hablar desde una perspectiva funcional del fenómeno -0 de la técnica- de la adposición, y esto porque en las lenguas se dan preposiciones y postposiciones, y no necesariamente con carácter exclusivo dentro de cada lengua:

Existen dos tipos de adposiciones: preposiciones y posposiciones. Las primeras dominan en las lenguas indoeuropeas. En las lenguas aglutinantes dominan las posposiciones. (Moreno 1987: 96)

Lo que quiere decir que en español, por ejemplo, también hay postposiciones. En el lugar citado se recogen: «nfo arriba», «rio abajo». Pero también los alemanes: «mir gegenuber», «Meiner Meinung nach», etc. $Y$ podriamos añadir: «carretera adelante», «mucho tiempo después», «longtemps apres», «beaucoup de siecles auparavant', «a long time before», etc. etc. Ahora bien, la mención de estos fenómenos suscita dos interrogantes:

1) Cómo se analizan sintagmas como los citados dentro de la teoría de la $X$ con barra, $\mathbf{y}$ cómo se analizan en general los adverbios y las locuciones adverbiales, y tambien los ablativos absolutos. Sobre esto hay que decir que el análisis debe preceder a la teoría, y no realizar el etiquetado de sintagmas a conveniencia de la teoría. Piénsese, por ejemplo, que el complemento de la cabeza de un sintagma postpositvo, valga la expresión, iría a la izquierda en lenguas right-branching como las citadas. Igualmente, hay que decir que las partes del sintagma no deben definirse solo a posteriori. No se debe llamar especificador o complementificador a todo lo que vaya a la izquierda de la cabeza para así evitar problemas. 
2) Algunos dirán que los ejemplos aducidos son en su mayor parte casos periféricos. Sobre esto hay que decir también que la delimitación de centro frente a periferia no puede hacerse sólo a conveniencia de la teoría .

En cualquier caso, resumamos diciendo que la formulación implícita en la teoría de la $\mathrm{X}$ con barra es más ambiciosa de lo que parece a primera vista. Correspondería a un presupuesto cuya forma lógica, valga la expresión, correspondería a un enunciado de lógica relacional con dos variables ligadas con el cuantificador universal:

$$
\forall x \forall y\left(H y^{\wedge} \mathbf{P x y} \longrightarrow \mathrm{Sy}^{\wedge} \mathrm{Pxy}\right)
$$

Traduzcamos: Si x está en la relación 'ser cabeza del sintagma de tipo tal' con y, y éste es un sintagma de algún tipo, entonces se tiene la relación más general 'ser cabeza de sintagma'. Digamos: La cabeza de un caballo, si el caballo es un animal, es la cabeza de un animal, argumento sobre el que Augustus de Morgan llamó alguna vez la atención ${ }^{8}$. Serfa, en efecto, necesario preguntarse acerca de la construcción del concepto general de sintagma, porque es posible que el formalismo oculte que se ha construido la clase de sintagma.(dentro de la GBT, no dentro de otras teorías gramaticales) de una manera precisa, a saber, a partir de cuatro clases distintas relacionables con cuatro partes de la oración: el nombre, el adjetivo, el verbo y la preposición'. En el contexto de la GBT no es el caso que la clase sintagma se haya dividido en cuatro subclases diferentes, sino que cuatro clases de estructuras diferentes se han unido formando una clase que comprende a todas ellas ${ }^{10}$. Esta es cuestión central de la teoría de la ciencia linguística, la de la constitución de las categorías de la misma, pero no podemos entrar aquí en ella. Nos limitamos a subrayar, una vez más, que el desarrollo de la teoría se mueve siempre en la dirección de una mayor generalidad de las clases de referencia que se van constituyendo.

- Garrido (1991), entre muchos otros manuales, presenta y discute este conocido argumento de logica de relativos.

- Lo que podía llevar a pensar según lo antes dicho que, desarrollada la gramática generativa en otro contexto linguístico, tal vez habría partido de distintas partes de la oración. Por ejemplo, no es inverosímil que la distinción entre sintagma adjetival y sintagma nominal no se hubiera producido en una lengua cuyos gramáticos no hubieran concedido estatuto aparte al adjetivo.

10 Sin embargo, el de los sintagmas prepositivos parece bastante peculiar. Ademas de las cuestiones citadas por Fanselow a las que hicimos referencia más arriba, recuerde el lector el tratamiento excepcional dado a estos sintagmas cuando se se trata de morfologia o en la modema fonología prosódica. Igualmente, el estudio de patologias del habla señala una diferencia entre este tipo de sintagmas y los demás. Para un breve resumen y una someras si bien útiles indica gráficas, véase Nespor, M. y Vogel, I. (195-96:1986 1994). 
Nuestro planteamiento ingenuo del parámetro y los fenómenos responde pues a la pluralidad y el proceso de totalización que la definición de todo parámetro lleva implícitos. Por lo que a la adquisición de una lengua hace, fijar el valor de un parámetro, en la formulación más general, es algo que, salvo la introducción de alguna otra ley o principio no contradicho por resultado empírico en contrario, requeriría la consideración de la exposición del infante a estímulos de naturaleza diversa.

Los estímulos que recibe, las proferencias que oye quien está aprendiendo una lengua, han considerarse desde la perspectiva del aprendizaje como evidencias de regularidades gramaticales ${ }^{11}$. Diferentes son los tipos de evidencia que puede encontrar aquel que aprende una lengua, sepa o no otra ${ }^{12}$ :

1) La evidencia positiva: exposición del aprendiz a proferencias de oraciones que ejemplifican fenómenos controlados por uno o más parámetros. En otras palabras, oraciones cuya estructura se determina según los valores adoptados por uno o más parámetros.

La evidencia negativa, que puede ser:

2) Directa: correcciones metalinguísticas efectuadas por otra persona. $\mathrm{Pa}$ rece, empero, verosímil que las correcciones para una primera lengua sean más bien de fenómenos relacionados con la periferia. Y sólo en el caso del aprendizaje de una segunda o tercera lengua, abunden las correcciones de este tipo referidas al núcleo de la gramática.

3) Indirecta: Simplemente, no se oyen oraciones que ejemplifiquen posibilidades gramaticales que no son del idioma, salvo errores, incoherencias, etc. No parece que aquí valga el quiasmo de que esta evidencia negativa es algo así como la negación de la evidencia. Se trata más bien de la ausencia de evidencia. Las opiniones en contra de la evidencia negativa indirecta, o simplemente evidencia negativa abundan. Más abajo, al final de este apartado citaremos algunos trabajos que muestran el desarrollo de la discusión.

El parámetro pro drop regula, entre otras $\operatorname{cosas}^{13}$, la necesidad de la aparición en la estructura-s de un sujeto: $I$ go es gramatical en inglés. $\mathrm{Ni}$ *go ni

11 Esto es, a partir de unos casos, tal vez pocos, se percibe o se postula una regularidad.

12 Seguimos el seminal Chomsky (1981: 8 y ss.) En otros lugares varía algo la terminología.

13 Véase la nota 7 más arriba. La dependencia entre los datos y los conceptos es asunto complejo. Piénsese en el filtro that-huella. La categoría vacta delante del verto no está regida propiamente, pero este es un resultado teórico que, sin perjuicio de su corrección, sólo puede describirse una vez que la teorfa se ha desarrollado. Como hecho gramatical - la categoria vacía no se rige si aparece por medio la conjunción that - es distinto del hecho gramatical «no incluir la conjunción that en estructuras como 'who do you think I saw yesterday?w'. Insistimos en que consideraciones de este tipo nos hacen sentimos autorizados para plantear la cuestión en los términos en que lo hacemos. 
* go I lo son. En cambio, Yo voy, Voy y Voy yo son tres posibilidades perfectamente gramaticales en español. Se tiene entonces un doble valor del parámetro, sin perjuicio de que, según lo dicten las características de nuevas lenguas investigadas, se haga necesario añadir algún posible valor más.

En el caso del parámetro mencionado y tomando, por un lado, una lengua como el inglés o el francés, y por el otro una como el español o el italiano se observa que las estructuras posibles que afecten a $\mathrm{SN}_{1}-\mathrm{S} \mathrm{V}$ en la forma fonética en las primeras son también posibles en las segundas, pero no a la inversa. Se habla entonces del principio del subconjunto. Parecerfa intuitivamente claro que las estructuras posibles en inglés son un subconjunto de las posibles en español. A propósito de la fijación del valor del parámetro, se ha sostenido que el infante avanza una primera hipótesis -como puede comprobarse, la metáfora del niño gramático puede realizar grandes progresos alegóricos-que consistiría en tomar el valor del parámetro correspondiente sólo al subconjunto (I go - yo voy). Entonces, en español la evidencia positiva de voy y voy yo cambiaría el valor. En inglés esto no sucedería, por cuanto si alguna evidencia hay de formas equivalentes es precisamente negativa. De aquí a sostener una fijación de valores por defecto, ya programada en el recién nacido hay un paso. Esto nos situaría ante el panorama de que unas lenguas estarían posiblemente más cercanas a la GU que otras ${ }^{14}$. La GU se definiría por sus principio, sus parámetros y por unos ciertos valores, lo que -pese a asumirse tácitamente por muchos autores- contradice gravemente la misma definición de GU. Ha habido cierta polémica sobre cuál de dos es el valor por defecto de un parámetro en el caso del pro-drop. Nos limitaremos aquí a observar que el referido principio del subconjunto se encuentra con alguna evidencia contraria, la de proferencias como *go en lugar de you go por niños pequeños. Por eso mismo, en Hyams (1986) se sostiene la fijación de valores opuesta. Ahora bien, esto sólo probaría que un niño pequeño tiene intención comunicativa, una cierta competencia pragmática y, en cambio, no domina aún la morfosintaxis. Puede verse también Hulk ( 1987), Manzini y Wexler (1987) y en relación con el aprendizaje de segundas lenguas White (1986). Esta autora ha defendido en, por ejemplo, White(1991a), (1991b) y (1992) el papel de la evidencia negativa frente a, por ejemplo, Schwartz y Gubala-Ryzak (1992). Digamos finalmente que el Principio del Subconjunto sigue dando lugar a no pocas polémicas. Puede verse, en contra del mismo, Kapur et al. (1993), y a favor, en réplica a éste último, Wexler (1993).

14 Hay que reconocer que se ha buscado evidencia empirica $\longrightarrow$ por mejor decir, corroboración- del principio en procesos de adquisición en general, y también en sujetos con deficiencias auditivas o de otra índole. No entraremos aquí en el examen de este tipo de investigación. 


\section{UNA DEFINICIÓN ALTERNATIVA DE PARAMETRO}

La noción de parámetro ha recibido críticas desde puntos de vista muy diversos (véase el ya citado Fanselow ed. (1993) para críticas desde posiciones muy cercanas a la GBT ortodoxa). Nuestra crítica particular se inicia con una consideración que para algunos resultará impertinente $o$, al menos, interesada. Nos parece que una buena parte del atractivo externo de la GBT reside en que, siendo sedicentemente una teoría de principios y parámetros, viene a prometer gramáticas que informacionalmente ocupen muy pocos bits, pero que sean suficientes para describir la GU y cualquier gramática nuclear particular. Sin embargo tal ambición teórica se acompaña de límites muy claros en la capacidad de la GBT para tratar con las cuestiones de la variabilidad linguística real, empírica. Y no porque la combinatoria que pueda surgir de un número reducido de parámetros sea insuficiente (por ejemplo con veinte parámetros con dos valores posibles cada uno y que puedan ajustarse independientemente se obtendría $2^{20}=1048576$ gramáticas nucleares nada menos), sino porque ese número reducido de parámetros se postula en el vacío, pero éstos y sus valores siguen sin conocerse. La alternativa heurística, nos parece, es -sin resumir de antemano la información precisa que paramétricamente definiría cada gramática- intentar a posteriori y empíricamente encontrar qué parámetros, si acaso, definen los fenómenos que se consideren centrales, y qué valores, entre los aprioristicamente posibles, se dan en las lenguas. Dentro de ese esquema los resultados de la GBT podrian servir de guía para la investigación, como razonaremos más abajo. En este sentido, se dirfa que la tarea de definir el conjunto de las lenguas téricamente posibles, desde los comienzos de la investigación chomskiana ha ido reduciendo cada vez más el campo. Sin embargo, cuando el "programa» - por decirlo en términos lakatianos-generativista ha construido gramáticas de lenguas existentes se ha quedado siempre por debajo de las necesidades impuestas por la pluralidad de las lenguas del mundo.

Que un parámetro sea tal es porque puede tomar un valor entre varios. Cuál sea el dominio de aplicación del parámetro y cuáles sean aquellos valores es lo que se trata de ver. Si un enfoque deductivo presenta los problemas señalados, tratemos de desarrollar un enfoque inductivo ${ }^{15}$, que tome como punto de

15 Por ser un enfoque empirico e inductivo la técnica que expondremos no puede partir principalmente de conceptos teóricos, esto es, tomando los conceptos téricos como datos. Intentaremos acercarnos lo más posible a los hechos, aunque ciertamente éstos lo son desde una teoria dada. En concreto, el carácter de la técnica «numérica» que presentamos más abajo no puede realizarse con, por ejemplo, los dominios de los que se habla en la cita que hemos hecho de Fanselow ( 1993), porque al definirlos se sesga la información empírica que intentamos preservar. 
partida las realizaciones lingüísticas presentes, aceptables y gramaticales para cada lengua. Es decir, aquellos datos que aparecen como casos de evidencia positiva tanto para el lingüista como para el hablante.

Aquí surge inmediatamente una cuestión. Por más pobre que sea el estímulo que el niño recibe (insuficiente para cualquier modelo conductista de aprendizaje, al decir de Chomsky, como ya recalcamos), es necesario postular un grado de evidencia positiva: si no se oye una lengua, una lengua de verdad y no la inaudible GU, no se aprende a hablar, es decir, a hablar una lengua que es la única manera conocida de hablar; y además, pasado cierto tiempo, parece deteriorarse hasta su pérdida la facultad de aprenderla. La cuestión hace referencia a qué inputs linguísticos son necesarios y suficientes para la asignación de un valor a un parámetro dado. Por lo que hace a la evidencia positiva nos encontraríamos con las siguientes posibilidades:

- Evidencia positiva de un fenómeno regulado por un parámetro, sin contar la intervención de uno o más principios. Por mejor decir otra vez: evidencia positiva de instanciaciones de una subclase de la clase de referencia del parámetro.

- Evidencia positiva de varios o todos los fenómenos regulados por el parámetro. O sea: de todos los tipos distinguibles en lo que puede llamarse el dominio del parámetro: todas las clases de cabezas, por ejemplo en el caso del Head Parameter.

- Evidencia positiva de fenómenos regulados por algunos o todos los parámetros de la lengua.

De hecho, la descrita en último lugar describe con realismo la situación que se da en el aprendizaje de una primera lengua, pero el aprendizaje de una segunda lengua, especialmente, en un contexto escolar formal, puede desviarse de esta situación, o bien puede registrar algún desequilibrio.

Además de los tipos de evidencia positiva enumerados, es importante también la cuestión acerca de qué grado de estímulo señala el umbral necesario para el ajuste de un parámetro. Y, en el caso del aprendizaje de una segunda lengua, aceptando de momento ${ }^{16}$ que el parámetro adquiere un nuevo valor $o$, mejor, la posibilidad de alternar, si es el caso, entre dos valores distintos, ¿qué es lo que sucede? Finalmente, ¿qué papel juega la evidencia negativa indirecta, si juega alguno?

Vamos a defender la hipótesis, admitiendo que el proceso descrito por la teoría es psicolinguísticamente adecuado, de que no existe un valor por defecto

16 La adquisición y el aprendizaje de una segunda lengua pueden tener lugar a través de procesos que impliquen o que no impliquen a la Gramática Universal. Es ésta una cuestión en disputa. Liceras (1992) es una inmejorable exposición. 
para ningún parámetro. Igualmente sostendremos que el valor final de un parámetro define y resume una información tan compleja acerca de la lengua en cuestión de la que se trate que más vale, no condensar, al menos en principio, la información aportada por el parámetro.

Sea el parámetro del pro-drop, nos limitamos por tanto a FLEX ${ }^{17}$ como cabeza, es decir, a la cuestión del sujeto nulo o null subject. Tras examinar diversas lenguas se concluye provisionalmente que el parámetro puede tomar dos valores, + y -. Digamos también que el rótulo ' + ' corresponde a una situación como la del español y el ' $\because$ ' a la del inglés.

Entonces, sostenemos que en GU el parámetro no está fijado por defecto en - ni en + , sino en un estado 0 , cuyo sentido se comprenderá por lo dicho a continuación. En una lengua de valor + para el fenómeno de la disposición relativa de $\mathrm{SN}_{1}$ y SV en la forma fonética, el valor del parámetro se definiría por una tripleta (SNl-SV(+), 0-SV-O(+), SV SN1(+)) y en una lengua - por (SN1-SV(+), 0-SV-O(0), SV-SN1(0)). En GU, la tripleta sería algo así como (SN1-SV(0), 0-SV-0(0), SV-SN1(0)). En una lengua - de $(0,0,0)$, que describiría a la GU, se pasaría a $(+, 0,0)$. A $(+,+,+)$ se llegaría en una lengua + . Así el rótulo ' $t$ ' resumiría una información más compleja, por ejemplo, $(+,+,+)$. El rótulo ' - , resumiría $(+, 0,0)$. Obsérvese que estas tripletas resumen información empírica, sin un compromiso teórico muy elevado, esto es, no se utilizan demasiadas hipótesis sobre la naturaleza del fenómeno del sujeto nulo.

En nuestra opinión este modelo se compadece mejor con los presupuestos de la GBT que el principio del subconjunto, si bien es cierto que pagando el alto precio de convertir un principio clasificatorio valioso - un bit de información $+/$ - nos dice mucho de la lengua - en un principio más prolijo y más caro informacionalmente. Por otro lado, sería posible acoplar esta propuesta a un modelo conexionista de aprendizaje con relativa facilidad, lo que tendría, obiter dictum, su interés cuando en un proceso de aprendizaje de una segunda lengua se hubiera de cambiar el valor de un parámetro: no se pasaría de $0 \mathrm{a}+6 \mathrm{a}-$, sino de $+\mathrm{a} 0$ o de $0 \mathrm{a}+$ en cada una de las posiciones de la, en este caso, tripleta. El modelo admitiría una métrica donde la distancia entre lenguas se definiese de acuerdo con una expresión del tipo:

$$
d=\sqrt{ }\left[\left(a_{i}^{*} b_{i}\right)^{2}+\left(a_{j}^{*} b_{j}\right)^{2}+\ldots\right] / \max _{1-n}\left(\min \left(a_{i}, b_{i}\right)\right)
$$

17 FLEX traduce a INFL. Como CONC, concordancia es abreviatura usual traduciendo a AGR, agreement. 
donde $a * b=0$ si $a=b ;$ y $a * b=1$ si $a \neq b$, siendo $a, b$ los valores en cada posición de la tripleta o, en el caso más general, de la n-tupla considerada El denomina-

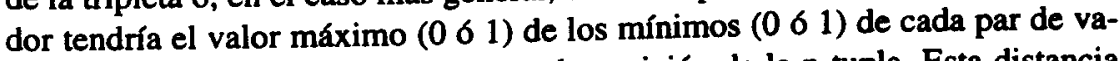
lores formados por los que ocupan cada posición de la n-tupla. Esta distancia daría verosímilmente alguna indicación acerca de la dificultad relativa de una lengua para el hablante de otra. Sin embargo, no tendría sentido la distancia entre GU y el núcleo de una lengua real cualquiera porque el denominador de la fórmula sería 0 , y ése sería el único caso en que lo fuera.

Podemos exponer, ampliando lo dicho hasta ahora, nuestra propuesta metodológica de manera sintética de la siguiente manera:

1) Se supone que cada parámetro se aplica a un dominio plural. En el prodrop todas las construcciones que venimos citando y recapitulamos más abajo; o en el head parameter, todas las clases de sintagmas, etc..

2) Cada dominio se divide en sectores, cada uno de ellos correspondiente a, siguiendo con el mismo ejemplo, un tipo de cabeza.

3) El sector se definiría por una n-tupla. N sería el número máximo de estructuras gramaticalmente admisibles.

4) Un parámetro con su valor fijado se definiría por una serie de n tuplas, tantas como sectores. En GU, un parámetro vendría definido con una fórmula o matriz como por ejemplo:

$[(0,0,0)(0,0)(0,0)]$

En una lengua dada, podría ser algo como:

$[(+,+,+)(+,+)(+,+)]$

y en otra algo como:

$[(+, 0,0)(+, 0)(+, 0)]$

El valor + o - del que hablábamos antes vendría a resumir una y otra expresión si, de hecho, sólo se dieran dos combinaciones.

El parámetro pro-drop regula hasta otros cuatros fenómenos distintos del mencionado del sujeto nulo, lo que tal vez implique que se aplica a más cabezas que a FLEX en el sentido del nudo equivalente a la cabeza de la oración princi$\mathrm{pal}^{18}$. Como se indicó antes, cabe preguntarse qué evidencia positiva basta para

18 Lo que se refiere a la siguiente estructura: [N"[I-V"]I']S=I". 
la fij ción del parámetro ¿Se necesita evidencia positiva de todos los fenómenos amparados bajo el parámetro en cuestión?. ¿Basta con algunos? $O$ bien, ¿existen combinaciones óptimas de estímulos que requieren un nivel de input menor de algunos fenómenos?

$Y$ otra vez, ¿qué papel juega la evidencia negativa indirecta? Por ejemplo puede pensarse que la inexistencia de un cierto tipo de in lleva a una especie de fijación del 0 en algún lugar de la fórmula descriptiva del parámetro, determinando así, al menos parcialmente, el valor adoptado por el parámetro. Esto ciertamente correspondería a una concepción dinámica de este tipo de evidencial $^{19}$. Más abajo daremos nuestra contestación a estas preguntas.

\section{Contrastación de La GBT y SEgundas Lenguas}

Si se admite que la GU juega algún papel en el aprendizaje y en la adquisición de una segunda lengua, sea directamente, sea a través de los valores de la gramática nuclear de la lengua ya conocida, y dado que una experimentación con los inputs linguísticos recibidos por el infante son difícilmente mensurables y manipulables, cabe pensar que la investigacion del aprendizaje y la adquisición de una segunda lengua no sólo aportaría evidencia acerca de la relación, mediata o inmediata, entre GU y la lengua de que se trate, sino también acerca del nivel de input, dicho de manera pedestre pero expresiva, del número de ejemplos de cada estructura necesarios para una variación en el valor de un parámetro, o para la duplicación de los valores del parámetro.

Si la GU en efecto interviene en el proceso, lo que parece equivalente a decir que los procesos implicados en la producción o comprensión de la segunda lengua son similares a los que lo son en el caso de la materna, puede comprenderse que la teoría tendrá en el caso de la adquisición o aprendizaje de una segunda lengua la piedra de toque ineludible. No es de extrañar que los alineamientos de unos y otros autores en torno a la intervención o no de la GU sean de carácter radical.

Es posible desarrollar diversos tipos de tests empíricos dirigidos a elucidar este asunto. Ha de hacerse la salvaguardia de que este tipo de tests pueden aportar algún criterio decisorio acerca de distintos modelos dentro del marco

19 Al respecto de ésta dice Chomsky: «a not unreasonable acquisition system can be devised with the operative principle that if certain structures or rules fail to be exemplified in relatively simple expressions, where they would be expected to be found, then a (possibly marked) option is selected excluding them in the grammar, so that a kind of «negative evidencem can be available even without corrections, adverse reactions, etc. (1981:9). 
GBT, pero quizá no permitan tan fácilmente, en cambio, decidir entre la GBT y otros marcos teóricos rivales ${ }^{20}$.

El problema metodológico central es el de neutralizar los múltiples factores que interfieren en los procesos de adquisición y aprendizaje. Las variables seleccionadas deberán estar relacionadas con los parámetros establecidos, aunque sean tentativamente por la teoría, $Y$ además de ellas, se deberá seleccionar qué tipos de inputs, qué tipos de evidencia se manejan. Se comprende que además del debido tratamiento estadístico de los resultados, fueran éstos cuales fueren, y con anterioridad se deberán establecer protocolos muy rígidos para el desarrollo de los experimentos.

Otro aspecto a tener en cuenta es que tales experimentos, desarrollados con sujetos que son de hecho estudiantes de una segunda lengua, se insertan dentro del largo y complejo proceso del aprendizaje. Deberán diseñarse, por tanto, de tal modo que, en la medida de lo posible, se aíslen de las etapas anteriores del proceso general. Igualmente, deberá tenerse en cuenta que los incrementos en las competencias demostradas a través de tests son generalmente decrecientes a partir de cierto nivel, de manera que hay que llevar a cabo una normalización de los resultados.

\section{LA CUESTIÓN DE LA INTERACCIÓN ENTRE PARÁMETROS}

Planteamos ahora la que nos parece una cuestión capital. De la misma manera que todos los módulos del modelo son necesario para realizar descripciones estructurales con capacidad explicativa, habría de pensarse que los valores que toman los parámetros en una lengua determinada no son arbitrarios cuando se consideran en conjunto.

Así, por ejemplo, es obvio que el parámetro pro-drop tiene que ver con la riqueza de CONC, de las desinencias personales de los verbos, aunque algunas lenguas contradicen este aserto. Se dice, y con razón, que salvo cuestiones pragmáticas, de focalización o énfasis, el yo de yo $c$ o mo es redundante en español por cuanto la desinencia ya informa acerca del sujeto de la oración. Se ha llegado incluso a sostener que habría que identificar SNI con esa desinencia. No sería 6 ptimo, desde el punto de vista de la eficacia comunicativa que el parámetro se fijara en español al revés.

20 Esto no es algo especialmente misterioso. Se trata de que el modelo o la teoría se definen sólo en parte por el resultado de la experimentación. Cabe la posibilidad, de hecho ya real, de que las posiciones de la GBT se concilien con los puntos de vista de la gramática funcional. Véanse las consideraciones finales de este trabajo. 
El panorama es pues, en resumen, que la fijación de los valores de los parámetros ha de aproximarse a algún tipo de óptimo de eficacia comunicativa $o$, si se quiere evitar un lenguaje de apariencia determinista, habría lenguas mejor parametrizadas que otras, aunque puede que las existentes fueran todas óptimas. Sin embargo, algunas posibles combinaciones de parámetros se desecharían por su carencia de funcionalidad en el sentido expresado, y no porque la GU las excluyera. Otras lenguas corresponderían a sistemas inestables y evolucionarían. Se podría suponer también que diversos niveles de descripción gramatical se realimentarían de manera que, por ejemplo, un determinado sistema fonológico planteara disfuncionalidades en compañía de un sistema morfológico o sintáctico con según qué características.

Una economía general de las lenguas, al estilo tal vez de la de Martinet, se dibuja en este contexto. Desde un punto de vista filosófico, semejante cosa parecería someter a las lenguas al reino de la necesidad, de una determinación distinta de la de la libertad del hablante, por decirlo en términos kantianos. Sin embargo, la perspectiva que avanzamos parece adecuada: los hablantes se encuentran con un sistema cuya arbitrariedad no llega al grado de que siempre se pueda libremente variar y de manera exitosa. El cambio no puede realizarse de manera estable si conduce a una gramática nuclear peor parametrizada en el sentido que acabamos de sugerir.

La evidencia negativa, a la luz de estas consideraciones, cobra un nuevo interés. En efecto, si existe una trabazón orgánica entre los aspectos controlados por los parámetros no todas las combinaciones de estímulos, por así decir, serán fácilmente aprendibles. Habría lenguas hipotéticas que no podemos fácilmente aprender, no sólo en el sentido chomskiano de que no se hallan previstas por la GU, sino en el sentido de que suponen una combinación de valores de parámetros ineficaz e incoherente. Una ausencia de evidencia positiva, esto es, una evidencia negativa al menos probabilísticamente a partir de cierto momento y dada una población aleatoria de inputs, reforzaría otra evidencia positiva que se viese ligada con ella. Sirva el ejemplo: no oír * go I reforzaría una evidencia positiva cuya naturaleza habría de determinarse empíricamente: tal vez se trataría de un fenómeno no controlado por el pro-drop. Además siempre sería posible llevar a cabo tests «de umbral» para las evidencias positivas de otros fenómenos controlados también por, en este caso, el pro-drop.

\section{OTRA AlTERNATIVA HEURISTICA}

Lo que hemos presentado en las secciones anteriores constituiría un método de contrastación empírica, sujeto a limitaciones como las apuntadas en la 
nota 16. Otros estudios de carácter distinto resultarian pertinentes. Puede verse que lo que hemos propuesto toma los parámetros de la GBT como hipótesis teórica que no sólo se busca contrastar, sino que -y esto es más importanteorienta la investigación. Si se toman los datos correspondientes a una gran variedad de lenguas para un parámetro dado - siempre en la medida en que los términos implicados tengan validez interlingüística - es razonable pensar que nos encontremos empíricamente con menos combinaciones que posibilidades abiertas téricamente. Aquí la estadística aporta diversas técnicas que pueden resultar de utilidad, si bien el extraer una variable oculta de un conjunto de datos - variable inexistente en muchos casos para los detractores de la aplicación indiscriminada del análisis multifactorial y técnicas similares- no es exactamente la contrastación de una teoría. Para una crítica asequible del abuso, que no del uso, de estos métodos el lector puede consultar Gould $(1981)^{21}$.

Si se nos permite una ilustración numérica, carente de todo rigor matemático y estadístico pero expresiva, diremos que lo que se buscaría sería algo así como lo siguiente. Sea un parámetro que domina m fenómenos. Si para cada fenómeno dado, caben tres posibilidades a priori, el número de soluciones gramaticales al fenómeno será $\mathrm{N}=2^{3}=8$. En total, caben teóricamente para el parámetro un número de soluciones $P=N^{m}$. Si el número de soluciones observadas $\mathrm{Q}$ es considerablemente menor que $\mathrm{P}$-diciéndolo de manera muy poco rigurosa, insistimos- el planteamiento del parámetro será verosímil. Si eliminando uno de los fenómenos cubiertos por el parámetro la proporción entre casos reales y casos posibles decrece considerablemente, cabría pensar en que el fenómeno en cuestión no dependía del parámetro.

Naturalmente, no tendría sentido un número de combinaciones de casos mucho más grande que el habitual en la linguística comparativa y, en tal caso, habría que concluir que toda la investigación se hallaba mal encaminada. Además las clases que se obtuvieran y su variabilidad deberían poder explicarse dentro de la teoría.

Esta propuesta viene a plantear la comparación entre variabilidad real y variabilidad prevista no por la teoría sino por la mera combinatoria de casos obtenibles. Se trataría de reducir la teoría a un grado cero, si se nos permite la expresión, y estudiar si esa variabilidad prevista tiene lugar y en que proporción.

21 La cuestión aquí es cuando la estadística no viene a contrastar un modelo o una hipótesis sino a introducir nuevos términos en el campo. No parece improbable el encontrarse con una regularidad que no corresponda a ninguna realidad linguística. Incluso las regularidades pueden ser mal interpretadas y no corresponder a determinaciones lingüisticas, sino comunicacionales, fisiologicas, o de historia linguística. 
Sirva todo esto como ilustración no técnica - incluso no exenta de error- de la propuesta.

\section{Algunos comentarios finales}

- Nuestro interés se ha centrado en la discusión de la fijación del valor de un parámetro. Otra cuestión es la de si un parámetro con dos valores discretos, junto con otros parámetros y con algún principio, determina totalmente los fenómenos relacionados con ese mismo parámetro. En el caso del pro-drop, recapitulando observaciones que hemos dejado esparcidas a lo largo de nuestro artículo, diremos que para sư elucidación ${ }^{22}$ se precisa sólo de un principio y un parámetro. Los fenómenos de pronombre sujeto desaparecido o nulo, sujeto pospuesto libremente, infracción aparente del filtro that-huella (que huella), extracción del sujeto de una isla qu- y pronombre nulo reanudado, se acaban explicando mediante el principio de categoría vacía (que cada una de éstas ha de ir siempre propiamente regida o gobernada) y un parámetro de dos valores según la riqueza de CONC, esto es, si CONC expresa información acerca de género, número y persona. La pretensión declarada es abarcar muchos fenómenos bajo una explicación lo más económica posible. Cabe, empero, preguntarse si así se logran explicar los casos de lenguas como el chino mandarín, el japonés y el coreano, lenguas que permiten la desaparición del sujeto pero que carecen de CONC, que manifiestan cierta variabilidad fenómenica, por ejemplo, el sujeto puede suprimirse en chino, pero no puede postponerse. $O$, en hebreo moderno, en que depende la desaparición del sujeto del tiempo y la persona verbal ${ }^{23}$.

Por otro lado, es empíricamente evidente que la riqueza de la morfología verbal y nominal varía interlingüísticamente según una escala de muchos grados. Si el parámetro pudiera tomar más valores que dos, la teoría podría retomarse.

Si se intenta, como antes sugerimos, explicar casos como las de estas últimas lenguas mediante el recurso a un nuevo parámetro que interactuase con el de la riqueza de CONC, nos encontraríamos con que habíamos perdido la

22 Véase la asequible la síntesis ofrecida en van Riemsdijk y Williams (1986: 1990).

${ }^{23}$ Estas lenguas son mencionadas en la literatura GBT, pero la resolución del problema teórico que suponen no se resuelve. Eso sin contar con el problema descriptivo. Por ejemplo, cabe con razón preguntarse si el chino tiene sujeto. Pueden verse los trabajos reunidos en Li Ch. N. ed. (1976) para argumentar una respuesta negativa desde presupuestos funcionales. En nuestra opinión, estas cuestiones suponen un muy serio reto a la GBT. 
economía explicativa. Pero, lo que es más grave, postular en el vacío un nuevo parámetro parecería una solución demasiado ad hoc. Desde un punto de vista metateorético se estaría diciendo que la teoría completa lo explica todo, pero que ni nosotros ni nadie conoce esa teoría. Lo cual no excluye el hecho de que a priori una combinatoria de un número exiguo de principios y parámetros pueda abarcar la pluralidad empírica de las lenguas ${ }^{24}$.

En cualquier caso, y desde un punto de vista empírico, ya hemos dicho que creemos que la GBT debe hallar algún tipo de contrastación en los procesos de adquisición y aprendizaje de segundas lenguas. En ese contexto, junto con el de la investigación de la variabilidad real de las gramáticas de las lenguas existentes pueden obtenerse resultados significativos. A propósito de esta opción remanente de que unos fenómenos dados vengan determinados, además de por un principio y un parámetro de dos posibles valores, mediante la concurrencia de otros parámetros y otros principios, acabamos de ver que esto contradiría de principio una explicación como la expuesta para el pro-drop, pero empíricamente podría ser pertinente estudiar si basta la exposición a un fenómeno para fijar el nuevo valor del parámetro implicado en un caso de dos lenguas que se diferenciasen en un sólo parámetro. La cuestión de la interacción entre componentes - véase la nota número 5- de la que habla Chomsky (1981:9) es la que reaparece quí. Sin embargo, insistimos, no parece correcto dejar al futuro descubrimiento de nuevos principios la explicación de la casuística de una lengua cada vez que un fenómeno resulte difícil de abarcar por el modelo.

En Manzini ( 1993) tenemos un caso de esta naturaleza. Allí encontrará el lector un ejemplo de estudio en que se dice estudiar la interacción de dos parámetros: el Null Subject Parameter y el V-TO-I Parameter, donde I es, a los efectos, lo que antes hemos llamado INF y FLEX. Ahora bien, estos dos parametros no parecen sino casos particulares del Pro-drop Parameter. En efecto, Manzini (1993) ha de leerse sobre todo como un alegato en favor de versiones generalizadas de los parámetros:

Under the Lexical Parameterization Principle, the null subject parameter itself can be reduced simply to whether $I$ is or is not a head ... (151)

24 A priori, porque, como decimos, nada asegura que la teoría no sea otra cosa que un programa de investigación que haya eventualmente de ser abandonado. Por otro lado, no es imposible que las lenguas existentes sean menos que las posibles, y que la GU se construyera, erróneamente, de tal manera que excluyera a algunas de estas lenguas posibles. Este asunto se relaciona con el carácter contingente o necesario de nuestra capacidad linguística y de los idiomas históricamente dados. 
Esta manera de conjugar dos parámetros no es sino el ya visto proceso de ampliación del dominio de un parámetro.

- El cambio lingüístico encuentra en nuestra hipótesis un modelo al menos verosímil y encuadrable en la GBT. En efecto, un cambio lingüístico que afectase a la gramática nuclear no sería un simple y contundente cambio del valor de un parámetro, sino un cambio relativamente menor dentro de todos los valores que definen un parámetro dado. Podría haber situaciones inestables que evolucionasen hacia otras. Los valores de las matrices para una lengua históricamente estable podrían aportar alguna idea acerca de qué gramáticas son más estables. Desde luego, esta consideración, no hay que ocultarlo, es muy discutible, no sólo por las debilidades de la glotocronología, sino también por las variables externas que juegan en los procesos de cambio y evolución linguística.

- Nuestra hipótesis posee, como ya apuntamos, también una cierta verosimilitud o acomodo conexionista. Correspondería a un modelo conexionista en que el aprendizaje de un cierto ítem suponga cierto nivel de estímulo, pero con la posibilidad de que ese nivel se rebaje si existe cierto nivel de estímulo de otra clase, digamos, correspondiente a otro ítem. Igualmente, la presencia de un tercer tipo de estímulo podría incrementar el nivel de estímulo necesario para el aprendizaje de aquel ítem. La evidencia negativa indirecta consistiría en un no incrementar el nivel necesario de estímulo. Linguiísticamente, la situación sería la de una lengua comunicativa funcionalmente ineficaz, difícil de aprender, frente a una lengua eficaz, de adquisición más sencilla.

- La evidencia negativa directa o explicativa -pero también la positiva, y cualquier refuerzo- poseería también una virtualidad en la fijación de los niveles de umbral. En otras palabras, las reglas expresadas metalingüísticamente pueden integrarse en un modelo de las características reseñadas.

- El aprendizaje de una segunda lengua implica-dentro de un cierto modelo de aprendizaje ${ }^{25}$ - la posibilidad de alternar el valor de un parámetro con otro. Algo que nuestra definición de parámetro permite es la representación de estados intermedios y de algunos procesos de interferencia. En efecto, si se conoce una lengua para la que el valor de un parámetro se define por

$$
[(+,+,+)(+,+)(+,+)]
$$

y se trata de aprender otra, descrita respecto al parámetro por

${ }^{35}$ Existe amplia literatura al respecto. Véase como referencia de conjunto más cercana el ya citado Liceras (1992). 


$$
[(+, 0,0)(+, 0)(+, 0)]
$$

entonces pueden suponerse valores «intermedios», interlenguas podríamos aventurarnos a decir, representables como:

$$
[(+, 0,0)(+, 0)(+,+)] \text {, }
$$

Lo que daría una explicación del fracaso registrado en los intentos de intentar cambiar el valor de un parámetro exponiendo al aprendiz a un sólo tipo de fenómenos regidos por el parámetro. Salvo lo que indicasen investigaciones dirigidas según lo expuesto más arriba, resultados empíricos de esa suerte nos hablarían de que el aprendiz ha fijado el parámetro en un valor intermedio, tal vez inestable. Consideraciones similares cabe hacer sobre las lenguas pidgin. No se trataría de que la constitución de una lengua pidgin revelase la «verdad» de la GU, sino una situación de máxima funcionalidad dentro de un contexto cultural e interlingüístico dado.

- Puede parecer que lo que hacemos es desvirtuar la GBT. Ciertamente, la perspectiva que se deja entrever a lo largo de nuestras consideraciones puede ser calificada como funcionalista - funcionalista de gramática funcional, no de funcionalismo psicológico o filosófico-en el siguiente sentido: La noción de parámetro no responde a determinaciones innatas prima facie, o no sólo. Es preciso considerar una parametrización dada desde la perspectiva funcional, es decir, desde el punto de vista de su coherencia dentro del sistema lingüístico en que se inserta y a efectos comunicativos y pragmáticos. 0 , podría pensarse en un intento de síntesis entre el innatismo y el funcionalismo. Si el lenguaje ha evolucionado con la especie humana y ésta junto con otras, habrá que pensar en una eficacia funcional parcial de la facultad del lenguaje y de las lenguas. A esto lleva la concepción de Chomsky de la linguística como parte de la psicología, y esta como parte de la biología.

Por Eso, pensamos, cualquier decisión al respecto debe tener en cuenta que una inexcusable piedra de toque de un modelo lingüístico se encuentra en los procesos de adquisición y en el complemento del enfoque funcional.

\section{REFERENCIAS}

CHomsky, N. (1981) Lectures on Government and Binding. The Pisa Lectures, Foris

Publications, Dordrecht, Cinnamison.

Сномsкy (1991) 'A Minimalist Program for Linguistic Theory', Draft, MIT.

CooK, V. J. ed. (1986) Experimental Approaches to Second Language Learning, Pergamon, Oxford. 
Cook. J. V. (1988) Chomsky's Universal Grammar, Blackwell, Oxford, New York.

Fanselow, G. ed. (1993) The Parametrization of Universal Grammar, John Benjamins, Amsterdam / Philadelphia.

GARRIDO, M. (1991) Lógica simbólica, Tecnos, Madrid,.

GouLd, S. J. (1984) The Mismeasure of Man, Pelican Books, Harmondsworth .

HaEgeman, L. (1991) Introduction to Government \& Binding Theory, Blacwell, Oxford and Cambridge, U.S.A.

HulK, A. (1987) 'L'acquisition du français et le parametre pro-drop', en Kampersky, B. ed. (1987).

HyAms, N. (1986) Language Acquisiton and the Theory of parameters, Reidel, Dordrecht.

KAMPERSKY, B. ed. (1987) Etudes de linguistique fran,caise offertes a Robert de Dardel, Rodopi, Amsterdam.

KAPUR, Sh. et al. (1993) 'Universal Grammar and Learnability Theory: the Case of Binding Domains and the 'Subset principle", en Reuland, E. y Abraham, W. eds. (1993).

Kornal, A. Y Pullum, G.K. (199) «The X-Bar Theory of Phrase Structure», en Language, vol. 66. number 1, pp. 24-50.

L1, Ch. N. ed. (1976) Subject and Topic, Academic Press, New York.

LICERAS, J. M. (1992) La adquisición de segundas lenguas, Visor, Madrid.

Lopez GarciA, A. (1989) Fundamentos de lingüistica perceptiva, Gredos, Madrid.

ManzinI, R. (1993) 'Categories in the Parameters Perspective: Null Subjects and VTO-I.', en Reuland, E. y Abraham, W. eds. (1993)

MANZINI, R. y WeXIER, K. (1987) 'Parameters, binding theory and leamability', en Linguistic Inquiry, 18, 3, 413-44.

Moreno Cabrera, J. C. (1987) Fundamentos de Sintaxis General, Síntesis, Madrid. Nespor, M. Y VoGel, M., 1986:1994, La Prosodia, Visor, Madrid.

VAN RIEMSDUK, H. Y WILLIAMS, E. (1986: 1990) Introducción a la teoría gramatical, Cátedra, Madrid. Trad. de Introduction to the Theory of Grammar, M.I.T.

REULAND, E. Y ABRAHAM, W. eds. (1993) Knowledge and Language, Volume I, From Orwell's Problem to Plato's Problem, Kluwer, Dordrecht / Boston I London.

RIzzI, L.(1986) 'Null objects in Italian and the theory of pro', Linguistic Inquiry, 17, 501-558.

SCHWARTZ Y GUBALA-RYZAK ( 1992) 'Learnability and grammar reorganization in L2A: against negative evidence causing the unlearning of verb movement', Second Language Research, 8, 1, 138 .

SELLS, P. (1985) Lectures on contemporary Syntactic Theories, University oc Chicago Press, Chicago.

WeXLeR, K. (1993) «The Subset Principle is an Intensional Principle», en Reuland, E. y Abraham, W. eds. (1993). 
WHITE, L. (1986) 'Implications of parametric variation for adult second language acquisition: an investigation of the pro-drop parameter', en Cook, V. J. ed. (1986). WhITE, L. (1991a) 'Adverb placement in second language acquisition', Second Language Research, 7, 133-161.

WHITE (1991b) 'The verb-movement parameter in second language acquisition', Language Acquisition, 1, 337-360.

WhITE, L. (1992) 'On triggering data in L2 acquisition: a reply to Schwartz and Gubala Ryzak', Second Language Research, 8, 2, 120-137. 